Article

\title{
The Prevalence and Clinical Characteristics of TECTA-Associated Autosomal Dominant Hearing Loss
}

Rika Yasukawa ${ }^{1}$, Hideaki Moteki ${ }^{1}$, Shin-ya Nishio ${ }^{1,2}{ }^{(D}$, Kotaro Ishikawa $^{3}$, Satoko Abe ${ }^{4}$, Yohei Honkura ${ }^{5}$, Misako Hyogo ${ }^{6}$, Ryota Mihashi ${ }^{7}$, Tetsuo Ikezono ${ }^{8}$, Tomoko Shintani ${ }^{9}$, Noriko Ogasawara ${ }^{9,10}$, Kyoko Shirai ${ }^{11}$, Hiroshi Yoshihashi ${ }^{12}$, Takashi Ishino ${ }^{13}$, Koshi Otsuki ${ }^{14}$, Tsukasa Ito ${ }^{15}$, Kazuma Sugahara ${ }^{16}$ and Shin-ichi Usami ${ }^{1,2, *(\mathbb{D})}$

1 Department of Otorhinolaryngology, Shinshu University School of Medicine, 3-1-1 Asahi, Matsumoto 390-8621, Japan; yasukawar@shinshu-u.ac.jp (R.Y.); moteki@shinshu-u.ac.jp (H.M.); nishio@shinshu-u.ac.jp (S.-y.N.)

2 Department of Hearing Implant Sciences, Shinshu University School of Medicine, 3-1-1 Asahi, Matsumoto 390-8621, Japan

3 Department of Otolaryngology, National Rehabilitation Center for Persons with Disabilities, 4-1 Namiki, Tokorozawa 359-8555, Japan; ishikawa-kotaro@rehab.go.jp

4 Department of Otorhinolaryngology, Toranomon Hospital, 2-2-2 Toranomon, Tokyo 105-0001, Japan; abe3387@ybb.ne.jp

5 Department of Otolaryngology-Head and Neck Surgery, Tohoku University School of Medicine, 1-1 Seiryomachi, Sendai 980-0872, Japan; y-honkura@ktb.biglobe.ne.jp

6 Department of Otolaryngology-Head and Neck Surgery, Kyoto Prefectural University of Medicine, 465 Kagii-cho, Kyoto 602-8566, Japan; mihyogo@koto.kpu-m.ac.jp

7 Department of Otolaryngology-Head and Neck Surgery, Kurume University School of Medicine, 67 Asahi-machi, Kurume 830-0011, Japan; mihashi_ryouta@med.kurume-u.ac.jp

8 Department of Otorhinolaryngology, Saitama Medical University, 38 Morohongo, Moroyama 350-0451, Japan; ikez@saitama-med.ac.jp

9 Department of Microbiology, Sapporo Medical University School of Medicine, South-1, West-17, Sapporo 060-8556, Japan; shinto211@gmail.com (T.S.); ogasawara.n@sapmed.ac.jp (N.O.)

10 Department of Otorhinolaryngology, Sapporo Medical University School of Medicine, South-1, West-17, Sapporo 060-556, Japan

11 Department of Otorhinolaryngology-Head and Neck Surgery, Tokyo Medical University, 6-7-1 Nishi-shinjuku, Tokyo 160-0023, Japan; anko.anko25@gmail.com

12 Department of Medical Genetics, Tokyo Metropolitan Children's Medical Center, 2-8-29 Musashidai, Tokyo 183-8561, Japan; hiroshi_yoshihashi@tmhp.jp

13 Department of Otorhinolaryngology, Head and Neck Surgery, Hiroshima University Hospital, 1-2-3 Kasumi, Hiroshima 734-0037, Japan; tishino@hiroshima-u.ac.jp

14 Department of Otolaryngology, Fukushima Medical University, 1 Hikarigaoka, Fukushima 960-1295, Japan; kotsuki@fmu.ac.jp

15 Department of Otolaryngology, Head and Neck Surgery, Yamagata University Faculty of Medicine, 2-2-2 Iida-nishi, Yamagata 990-9585, Japan; tuito@med.id.yamagata-u.ac.jp

16 Department of Otolaryngology, Yamaguchi University Graduate School of Medicine, 1-1-1 Minamikogushi, Ube City 755-8505, Japan; kazuma@yamaguchi-u.ac.jp

* Correspondence: usami@shinshu-u.ac.jp; Tel.: +81-263-37-2666

Received: 1 August 2019; Accepted: 18 September 2019; Published: 24 September 2019

Abstract: TECTA is well known as a causative gene for autosomal dominant mid-frequency hearing loss observed in various populations. In this study, we performed next-generation sequencing analysis of a large Japanese hearing loss cohort, including eight hundred and twelve (812) subjects from unrelated autosomal dominant hearing loss families, to estimate the prevalence and phenotype-genotype correlations in patients with TECTA mutations. The prevalence of TECTA 
mutations in Japanese autosomal dominant sensorineural hearing loss families was found to be $3.2 \%$. With regard to the type of hearing loss, the patients with mutations in the nidogen-like domain or ZA domain of TECTA showed varied audiograms. However, most of the patients with mutations in the $\mathrm{ZP}$ domain showed mid-frequency hearing loss. The rate of hearing deterioration in TECTA-associated hearing loss patients and in the normal hearing Japanese control population were the same and regression lines for each group were parallel. We carried out haplotype analysis for four families which had one recurring missense variant, c.5597C $>\mathrm{T}$ (p.Thr1866Met). Our results revealed four different haplotypes, suggesting that this mutation occurred independently in each family. In conclusion, TECTA variants represent the second largest cause of autosomal dominant sensorineural hearing loss in Japan. The hearing loss progression observed in the patients with TECTA mutations might reflect presbycusis. The c.5597C $>$ T mutation occurred in a mutational hot spot and is observed in many ethnic populations.

Keywords: TECTA; non-syndromic hearing loss; DFNA8/12; autosomal dominant; prevalence; genotype-phenotype correlation; hearing progression; haplotype analysis

\section{Introduction}

Hearing loss (HL) is one of the most common sensory disorders. Congenital HL, in particular, affects one in 500-600 newborns and it is speculated that about $60 \%$ of cases are caused by gene mutations [1]. Currently, it is considered that approximately 120 genes are associated with sensorineural hearing loss (SNHL) [2], with non-syndromic HL accounting for 70\% of SNHL. Among them, autosomal recessive (AR) inheritance accounts for approximately $75 \%$ and autosomal dominant (AD) inheritance constitutes approximately 15-20\% of cases. Autosomal dominant sensorineural hearing loss (ADSNHL) tends to be post-lingual and progressive HL [3]. Some causative genes are characterized by typical HL patterns affecting only specific frequencies. Distinct mutations in one of the causative genes for ADSNHL, TECTA (DFNA8/12), are well known to be associated with mid-frequency HL, while other mutations are involved in high frequency HL. All kinds of mutations are found among various populations. TECTA is also a known genetic cause of autosomal recessive HL (DFNB21); however, most of the TECTA-associated HL is ADSNHL. It comprises 23 exons, and encodes a 2155 amino acid protein, $\alpha$-tectorin, which is non-collagenous glycoprotein that constitutes a major component of the tectorial membrane in the cochlea $[4,5]$. The tectorial membrane lies over the cochlear hair cells and is critical for both the mechanical amplification of acoustic stimulation by the outer hair cells and for its transmission to the inner hair cells, which are the genuine sensory cells [6]. Previous studies have reported that TECTA-associated ADSNHL showed characteristic mid- or high-frequency HL depending on the position of the mutations [5,7].

Recently, next-generation sequencing (NGS) has become commonly used for the genetic screening of SNHL. Although hundreds or thousands of variants may be identified by NGS, it is difficult to determine which variants are pathogenic. It is particularly difficult to prioritize potential causal variants within a family with AD inheritance in which only one particular variant leads to the production of the trait. Therefore, we should consider not only NGS analysis results but also the phenotypes of the subjects.

In this study, we (1) elucidated the prevalence of HL caused by TECTA mutations in Japanese ADSNHL patients, (2) examined the genotype-phenotype correlations for each domain, (3) analyzed the rate of HL deterioration for TECTA-associated HL patients, and (4) carried out haplotype analysis for one variant identified in four unrelated ADSNHL families to confirm whether the mutation occurred in a mutational hotspot or whether it was a founder mutation. 


\section{Materials and Methods}

\subsection{Subjects}

In this study, we enrolled eight hundred and twelve (812) Japanese subjects (age range: 0-86 years, mean age: 37.1 years) from unrelated ADHL families. This study cohort was taken from 67 otolaryngology clinics across Japan between June 2000 and May 2017. All subjects in this study suffered from non-syndromic HL without any other associated symptoms. A written informed consent was obtained from each proband and their family members prior to participation in this study. This study was approved by the Shinshu University Human Genetic Analysis Ethical Committee, as well as by the ethics committee of each participating institute. The study was conducted in accordance with the Declaration of Helsinki, with the protocol approved by the Ethics Committee of the Shinshu University School of Medicine No. 387, 4 September 2012 and No. 576, 2 May 2017. Clinical information was obtained for each proband and relatives from medical charts. In this study, we collected the following data: (1) pure-tone audiograms, behavioral audiometry, or auditory steady state responses (ASSR); (2) medical history, including onset of HL, progression, and episodes of vertigo; and (3) temporal bone imaging (computed tomography and/or magnetic resonance) if available.

Pure-tone average (PTA) was calculated by air conduction pure-tone audiometry or the ASSR or behavioral audiometry average threshold in the four frequencies $(0.5,1,2$, and $4 \mathrm{kHz})$. Hearing levels were classified based on the PTA of the better hearing ear: Normal hearing $<20 \mathrm{~dB}$; mild HL, 21-40 dB; moderate HL, 41-70 dB; severe HL, 71-95 dB; and profound HL >95 dB. We also calculated the mean hearing level of: (1) mid-frequency; $0.5-2 \mathrm{kHz}$, and (2) high-frequency; $4-8 \mathrm{kHz}$. Type of $\mathrm{HL}$ was classified based on the following: High-frequency HL, (2) - (1) $\geq+10 \mathrm{~dB}$; flat type HL, (2) - (1) < $\pm 10 \mathrm{~dB}$; and mid-frequency $\mathrm{HL},(1)-(2) \geq+10 \mathrm{~dB}$.

\subsection{Genetic Analysis and Pathogenic Interpretation}

In this study, we used amplicon re-sequencing for 68 genes previously reported as genetic causes of non-syndromic hereditary HL (Table S1). In brief, amplicon libraries were prepared using an Ion AmpliSeq ${ }^{\mathrm{TM}}$ Custom Panel (ThermoFisher Scientific, Waltham, MA, USA), and Ion AmpliSeq v2 plus kit (ThermoFisher Scientific) in accordance with the manufacturer's instructions. After amplicon library preparation, emulsion PCR, and next-generation sequencing were performed according to the manufacturer's protocol with Ion 200 sequencing kit (ThermoFisher Scientific) and Ion PGM sequencer (ThermoFisher Scientific) or Ion HiQ chef Kit (ThermoFisher Scientific) and Ion Proton sequencer (ThermoFisher Scientific). The detailed protocol has been described elsewhere [8].

The fastq files obtained were mapped against the human genome (build GRCh37/hg19) using the Torrent Mapping Alignment Program and the variants, including the SNVs, insertions, and deletions, were detected by Torrent Variant Caller plug-in software. The effects of each variant on the protein were analyzed using ANNOVAR software [9]. The variants affecting amino acid sequences (missense, nonsense, insertion/deletion, and splicing variants) were selected from the identified variants. The selected variants were then also filtered as less than $1 \%$ of (1) the 1000 genome database [10], (2) the 6500 exome variants [11], (3) the Human Genetic Variation Database (dataset for 1208 Japanese exome variants) [12], and (4) the 333 in-house Japanese normal hearing controls. To estimate the pathogenicity of missense variants, functional prediction software, included in the ANNOVAR software (Sorting Intolerant from Tolerant (SIFT) [13], Polymorphism Phenotyping (PolyPhen2) [14], Likelihood Ratio Test (LRT) [15], Mutation Taster, [16] and Mutation Assessor [17]) were used. Direct sequencing was used to confirm the candidate variants identified through the analysis pipeline. Segregation analysis of family members was also performed by direct sequencing.

The pathogenicity of the identified variants was evaluated in accordance with the American College of Medical Genetics (ACMG) standards and guidelines [18]. This system classified variants into five categories; pathogenic, likely pathogenic, uncertain significance, likely benign, and benign based on the various evidence. In addition, we referred to Inter Var when we evaluated variants [19]. 
A combined annotation dependent depletion (CADD) was also utilized to prioritize potential causal variants [20].

\subsection{Haplotype Analysis of the c.5597C > T Variant}

We conducted haplotype analysis for the c.5597C $>\mathrm{T}$ (p.Thr1866Met) variant identified in four unrelated ADSNHL families. The haplotypes within the $1 \mathrm{Mb}$ region surrounding position c.5597 were analyzed using a set of 11 single nucleotide polymorphisms (SNPs) (three sites upstream and eight sites downstream). To select the SNPs for haplotype analysis, Tag SNPs were searched by SNPinfo Web server of the National Institute of Environmental Health Sciences with the Hap Map JPN data set [21]. This analysis was performed using the direct sequencing method.

Data Availability Statement: The sequencing data are available in the DDBJ databank of Japan (Accession number: JGAS00000000201).

\section{Results}

\subsection{The Prevalence of TECTA Mutations in Japanese ADSNHL Patients}

We identified 32 variants in 35 out of 812 probands with ADSNHL. As shown in Tables 1 and 2, 26 variants were novel, and six variants were previously reported as causing ADSNHL. Of the 26 novel variants, 25 were missense and one was nonsense. The six previously reported variants were as follows; p.Asp197Asn, p.Thr562Met, p.His1400Tyr, p.Pro1791Arg, p.Thr1866Met, and p.Arg1890Cys [5,22-24].

We next categorized these variants based on the ACMG criteria and Inter Var [18,19]. Then, we compared the CADD Phred scores for each variant with the larger value of the minor allele frequency (MAF) in the ExAC database [25] or in the ToMMo 3.5KJPN database (Tohoku University Tohoku Medical Megabank Organization, Sendai, JPN) [26] to estimate the pathogenicity, as shown in the scatter plot in Figure S1. We further selected the candidate variants as follows; (1) VUS variants (indicated as blue points in Figure S1) identified in 10 probands were less likely to cause HL, thus we removed these variants from further analysis. (2) We removed the variants with high MAF and/or low CADD scores (under the dotted-line in Figure S1). (3) We removed the one nonsense variant c.4302C $>$ A (p.Tyr1434Ter) as a cause for DFNA8/12. Disease causing mechanism for TECTA -associated ADSNHL was thought to be a dominant-negative effect resulting from missense mutations [22,27]. Therefore, it is unlikely that this nonsense mutation is causative for ADSNHL. In addition, the proband (HL0644) with this mutation also carried another variant in the TECTA gene; c.4955G $>C$ (p.Asp1499His), which was categorized as VUS, suggesting autosomal recessive inheritance (DFNB21). Finally, we defined the remaining 22 variants as likely causative variants (Table 1). The pedigree and audiograms for each patient are shown in Figure 1 and the variants are listed in Table 1. The in silico prediction software scores for novel missense variants are shown in Table S2.

According to the above results, the prevalence of TECTA mutations in Japanese ADSNHL families was considered to be $3.2 \%$ (26/812 probands). Furthermore, when we limited our analysis to 'Likely pathogenic' variants, it was considered to be $1.8 \%$ (15/812 probands).

Among the identified variants, c. $5597 \mathrm{C}>\mathrm{T}$ was recurrent and identified in four unrelated ADSNHL families. Interestingly, the c.3995G >A variant identified in this study caused a p.Cys1332Tyr amino acid change. Kim et al. have reported the c.3995G $>$ T variant as pathogenic, which despite having a different nucleotide change, resulted in the same amino acid change [28]. 
Table 1. Summary of the clinical features and information of the likely pathogenic and candidate variants classified as VUS identified in this study.

\begin{tabular}{|c|c|c|c|c|c|c|c|c|c|c|c|c|c|c|c|c|c|}
\hline No. & $\begin{array}{c}\text { Sample } \\
\text { ID }\end{array}$ & Gender & $\begin{array}{l}\text { Onset } \\
\text { Age }\end{array}$ & $\begin{array}{l}\text { Age at } \\
\text { Genetic } \\
\text { Testing }\end{array}$ & Fluctuation & Progression & $\begin{array}{l}\text { Base } \\
\text { Change }\end{array}$ & $\begin{array}{c}\text { AA } \\
\text { Change }\end{array}$ & Domain & $\begin{array}{c}\text { Type of } \\
\mathrm{HL}\end{array}$ & Severity & $\begin{array}{l}\text { CADD } \\
\text { Phred }\end{array}$ & $\begin{array}{l}\text { ACMG } \\
\text { Criteria }\end{array}$ & $\begin{array}{l}\text { ACMG } \\
\text { Category }\end{array}$ & $\begin{array}{c}\text { MAF in } \\
\text { ExAC }\end{array}$ & $\begin{array}{l}\text { MAF in } \\
\text { ToMMo }\end{array}$ & Reference \\
\hline 1 & SNS5183 & $\mathrm{M}$ & 16 & 24 & Yes & No & $c .208 \mathrm{~A}>\mathrm{G}$ & p.N70D & & Flat & Mild & 24.0 & VUS & PM2, PP3 & - & - & This study \\
\hline 2 & 4238 & $\mathrm{~F}$ & Unknown & 49 & No & No & c. $494 \mathrm{C}>\mathrm{T}$ & p.T165I & NIDO & Mid & Mild & 32.0 & vUS & $\begin{array}{l}\text { PM1, PM2, } \\
\text { PP3 }\end{array}$ & 0.0000331 & 0.0004 & This study \\
\hline 3 & GNM5012 & $\mathrm{F}$ & 5 & 7 & Unknown & Yes & c. $589 \mathrm{G}>\mathrm{A}$ & p.D197N & NIDO & High & Moderate & 27.8 & $\begin{array}{c}\text { Likely } \\
\text { pathogenic }\end{array}$ & $\begin{array}{l}\text { PM1, PM2, } \\
\text { PP1, PP3 }\end{array}$ & - & - & Hildebrand, 2011 [22]. \\
\hline 4 & 4030 & M & 0 & 10 & No & No & c.605T $>\mathrm{C}$ & p.L202P & NIDO & Mid & Mild & 27.0 & $\begin{array}{c}\text { Likely } \\
\text { pathogenic }\end{array}$ & $\begin{array}{c}\text { PM1, PM2, } \\
\text { PP1, PP3 }\end{array}$ & - & - & This study \\
\hline 5 & HL2684 & $\mathrm{F}$ & 6 & 10 & No & Yes & c. $1816 \mathrm{~T}>\mathrm{G}$ & p.C606G & ZA (TIL1) & Mid & Mild & 22.3 & $\begin{array}{c}\text { Likely } \\
\text { pathogenic }\end{array}$ & $\begin{array}{l}\text { PM1, PM2, } \\
\text { PP1, PP3 }\end{array}$ & - & - & This study \\
\hline 6 & HL1389 & $\mathrm{F}$ & 6 & 34 & No & No & c.1898G $>\mathrm{T}$ & p.C633F & ZA (TIL1) & High & Moderate & 27.6 & VUS & $\begin{array}{c}\text { PM1, PM2, } \\
\text { PP3 }\end{array}$ & - & - & This study \\
\hline 7 & HL2875 & F & 7 & 23 & Yes & No & c. $2087 \mathrm{G}>\mathrm{A}$ & p.C696Y & ZA & Flat & Mild & 26.7 & vUS & $\begin{array}{c}\text { PM1, PM2, } \\
\text { PP3 }\end{array}$ & - & - & This study \\
\hline 8 & 2803 & $\mathrm{M}$ & 25 & 46 & No & No & c.2087G $>C$ & p.C696S & $\mathrm{ZA}$ & High & Moderate & 25.6 & VUS & PM1,PM2,PP3 & - & - & This study \\
\hline 9 & 4238 & $\mathrm{~F}$ & Unknown & 49 & No & No & c. $3043 \mathrm{G}>\mathrm{A}$ & p.E1015K & ZA (TIL2) & Mid & Mild & 26.4 & VUS & PM1,PM2,PP3 & 0.0000165 & 0.0004 & This study \\
\hline 10 & HL1942 & $\mathrm{F}$ & 59 & 77 & No & Yes & $c .3605 \mathrm{C}>\mathrm{T}$ & p.S1202F & $\begin{array}{c}\mathrm{ZA} \\
\text { (VWD3) }\end{array}$ & Flat & Profound & 24.1 & VUS & $\begin{array}{l}\text { PM1, PM2, } \\
\text { PP3, BS2 }\end{array}$ & 0.0000741 & 0.0001 & This study \\
\hline 11 & SNS5351 & $\mathrm{F}$ & 12 & 16 & No & No & $c .3850 \mathrm{C}>\mathrm{T}$ & p.R1284C & $\begin{array}{c}\mathrm{ZA} \\
\text { (VWD3) }\end{array}$ & High & Mild & 26.1 & $\begin{array}{c}\text { Likely } \\
\text { pathogenic }\end{array}$ & $\begin{array}{l}\text { PM1, PM2, } \\
\text { PM7, PP3 }\end{array}$ & - & - & This study \\
\hline 12 & HL3514 & M & 6 & 8 & No & No & $c .3850 \mathrm{C}>\mathrm{T}$ & p.R1284C & $\begin{array}{c}\mathrm{ZA} \\
\text { (VWD3) } \\
\end{array}$ & Flat & Mild & 26.1 & $\begin{array}{c}\text { Likely } \\
\text { pathogenic }\end{array}$ & $\begin{array}{l}\text { PM1, PM2, } \\
\text { PM7, PP3 }\end{array}$ & - & - & This study \\
\hline 13 & 2965 & M & 6 & 10 & No & No & c. $3995 \mathrm{G}>\mathrm{A}$ & p.C1332Y & $\mathrm{ZA}$ & High & Mild & 24.4 & $\begin{array}{c}\text { Likely } \\
\text { pathogenic }\end{array}$ & $\begin{array}{l}\text { PM1, PM2, } \\
\text { PP1, PP3 }\end{array}$ & - & - & This study \\
\hline 14 & HL0644 & M & 0 & 16 & No & Yes & c. $4495 \mathrm{G}>\mathrm{C}$ & p.D1499H & $\begin{array}{c}\mathrm{ZA} \\
\text { (VWD4) }\end{array}$ & Mid & Moderate & 29.7 & VUS & $\begin{array}{c}\text { PM1, PM2, } \\
\text { PP3, BS2 }\end{array}$ & - & 0.0006 & This study \\
\hline 15 & 3552 & M & 62 & 64 & Yes & Yes & c. $5372 \mathrm{C}>\mathrm{G}$ & p.P1791R & & Mid & Moderate & 22.5 & vUS & $\begin{array}{c}\text { PM2, PP5, } \\
\text { BP4 }\end{array}$ & 0.0002 & - & Hildebrand, 2011 [22] \\
\hline 16 & HL4169 & $\mathrm{F}$ & 6 & 13 & No & No & c. $5597 \mathrm{C}>\mathrm{T}$ & p.T1866M & $\mathrm{ZP}$ & Flat & Mild & 35.0 & $\begin{array}{c}\text { Likely } \\
\text { pathogenic }\end{array}$ & $\begin{array}{l}\text { PM1, PM2, } \\
\text { PM7, PP3, } \\
\text { PP5 }\end{array}$ & 0.00000824 & - & Sagong, 2010 [23]. \\
\hline 17 & HL3681 & $\mathrm{M}$ & 0 & 0 & No & No & c. $.597 \mathrm{C}>\mathrm{T}$ & p.T1866M & $\mathrm{ZP}$ & Mid & Moderate & 35.0 & $\begin{array}{c}\text { Likely } \\
\text { pathogenic }\end{array}$ & $\begin{array}{l}\text { PM1, PM2, } \\
\text { PM7, PP3, } \\
\text { PP5 }\end{array}$ & 0.00000824 & - & Sagong, 2010 [23]. \\
\hline 18 & HL0605 & $\mathrm{M}$ & 0 & 10 & No & No & c. $.5997 \mathrm{C}>\mathrm{T}$ & p.T1866M & $\mathrm{ZP}$ & Mid & Mild & 35.0 & $\begin{array}{c}\text { Likely } \\
\text { pathogenic }\end{array}$ & $\begin{array}{l}\text { PM1, PM2, } \\
\text { PM7, PP3, } \\
\text { PP5 }\end{array}$ & 0.00000824 & - & Sagong, 2010 [23]. \\
\hline 19 & 2271 & $\mathrm{M}$ & Unknown & 6 & Unknown & Unknown & c. $.5597 \mathrm{C}>\mathrm{T}$ & p.T1866M & ZP & Mid & Moderate & 35.0 & $\begin{array}{c}\text { Likely } \\
\text { pathogenic }\end{array}$ & $\begin{array}{l}\text { PM1, PM2, } \\
\text { PM7, PP3, } \\
\text { PP5 }\end{array}$ & 0.00000824 & - & Sagong, 2010 [23]. \\
\hline 20 & HL3110 & $\mathrm{F}$ & Unknown & 82 & No & Yes & c. $5668 \mathrm{C}>\mathrm{T}$ & p.R1890C & $\mathrm{ZP}$ & Flat & Severe & 34.0 & $\begin{array}{c}\text { Likely } \\
\text { pathogenic }\end{array}$ & $\begin{array}{l}\text { PM1, PM2, } \\
\text { PP3, PP5 }\end{array}$ & - & - & Plantinga, 2006 [5]. \\
\hline
\end{tabular}


Table 1. Cont

\begin{tabular}{|c|c|c|c|c|c|c|c|c|c|c|c|c|c|c|c|c|c|}
\hline No. & $\begin{array}{c}\text { Sample } \\
\text { ID }\end{array}$ & Gender & $\begin{array}{c}\text { Onset } \\
\text { Age }\end{array}$ & $\begin{array}{l}\text { Age at } \\
\text { Genetic } \\
\text { Testing } \\
\end{array}$ & Fluctuation & Progression & $\begin{array}{l}\text { Base } \\
\text { Change }\end{array}$ & $\begin{array}{c}\text { AA } \\
\text { Change }\end{array}$ & Domain & $\begin{array}{c}\text { Type of } \\
\text { HL }\end{array}$ & Severity & $\begin{array}{l}\text { CADD } \\
\text { Phred }\end{array}$ & $\begin{array}{l}\text { ACMG } \\
\text { Criteria }\end{array}$ & $\begin{array}{l}\text { ACMG } \\
\text { Category }\end{array}$ & $\begin{array}{l}\text { MAF in } \\
\text { ExAC }\end{array}$ & $\begin{array}{l}\text { MAF in } \\
\text { ToMMo }\end{array}$ & Reference \\
\hline 21 & 2787 & $\mathrm{~F}$ & 15 & 27 & Unknown & Yes & $c .5668 \mathrm{C}>\mathrm{T}$ & p.R1890C & $\mathrm{ZP}$ & Mid & Mild & 34.0 & $\begin{array}{c}\text { Likely } \\
\text { pathogenic }\end{array}$ & $\begin{array}{l}\text { PM1, PM2, } \\
\text { PP3, PP5 }\end{array}$ & - & - & Plantinga, 2006 [5]. \\
\hline 22 & HL0950 & $\mathrm{F}$ & 3 & 4 & No & No & c.5807T $>\mathrm{C}$ & p.L1936P & ZP & unspecified & Moderate & 25.4 & $\begin{array}{c}\text { Likely } \\
\text { pathogenic }\end{array}$ & $\begin{array}{l}\text { PM1, PM2, } \\
\text { PP1, PP3 }\end{array}$ & - & - & This study \\
\hline 23 & HL2094 & F & 3 & 8 & No & No & c. $5824 \mathrm{~T}>\mathrm{A}$ & p.Y1942N & $\mathrm{ZP}$ & Mid & Moderate & 32.0 & $\begin{array}{c}\text { Likely } \\
\text { pathogenic }\end{array}$ & $\begin{array}{c}\text { PM1, PM2, } \\
\text { PP1, PP3 }\end{array}$ & - & - & This study \\
\hline 24 & HL0916 & F & 6 & 38 & No & No & c. $5948 \mathrm{C}>\mathrm{T}$ & p.T1983I & $\mathrm{ZP}$ & Mid & Mild & 33.0 & vUS & $\begin{array}{c}\text { PM1, PM2, } \\
\text { PP3 }\end{array}$ & - & - & This study \\
\hline 25 & HL1026 & F & 0 & 4 & No & No & c.5987T $>\mathrm{A}$ & p.I1996N & $\mathrm{ZP}$ & Mid & Severe & 34.0 & $\begin{array}{c}\text { Likely } \\
\text { pathogenic }\end{array}$ & $\begin{array}{l}\text { PM1, PM2, } \\
\text { PP1, PP3 }\end{array}$ & - & - & This study \\
\hline 26 & HL3056 & F & 4 & 32 & Yes & Yes & c. $5999 \mathrm{G}>\mathrm{T}$ & p.G2000V & $\mathrm{ZP}$ & Flat & Moderate & 26.2 & vUS & $\begin{array}{c}\text { PM1, PM2, } \\
\text { PP3 }\end{array}$ & - & - & This study \\
\hline 27 & HL2846 & M & 2 & 71 & No & No & c. $6183 \mathrm{G}>\mathrm{T}$ & p.R2061S & & Mid & Severe & 24.4 & VUS & $\begin{array}{l}\text { PM2, PP1, } \\
\text { PP3 }\end{array}$ & - & - & This study \\
\hline
\end{tabular}


Table 2. Summary of the clinical features and information of the plausible benign VUS and likely benign variants identified in this study.

\begin{tabular}{|c|c|c|c|c|c|c|c|c|c|c|c|c|c|c|c|c|c|}
\hline No. & $\begin{array}{l}\text { Sample } \\
\text { ID }\end{array}$ & Gender & $\begin{array}{l}\text { Onset } \\
\text { Age }\end{array}$ & $\begin{array}{l}\text { Age at } \\
\text { Genetic } \\
\text { Testing } \\
\end{array}$ & Fluctuation & Progression & $\begin{array}{l}\text { Base } \\
\text { Change }\end{array}$ & $\begin{array}{c}\text { AA } \\
\text { Change }\end{array}$ & Domain & $\begin{array}{c}\text { Type of } \\
\text { HL }\end{array}$ & Severity & $\begin{array}{l}\text { CADD } \\
\text { Phred }\end{array}$ & $\begin{array}{l}\text { ACMG } \\
\text { Criteria }\end{array}$ & $\begin{array}{l}\text { ACMG } \\
\text { Category }\end{array}$ & $\begin{array}{l}\text { MAF in } \\
\text { ExAC }\end{array}$ & $\begin{array}{l}\text { MAF in } \\
\text { ToMMo }\end{array}$ & Reference \\
\hline 1 & HL0150 & M & Unknown & 60 & No & Yes & c. $842 \mathrm{~T}>\mathrm{C}$ & p.V281A & $\begin{array}{c}\mathrm{ZA} \\
\text { (VWC) }\end{array}$ & High & Moderate & 10.5 & vUS & $\begin{array}{c}\text { PM1, PM2, } \\
\text { BP4 }\end{array}$ & - & - & This study \\
\hline 2 & SNS5496 & $\mathrm{F}$ & 50 & 57 & No & Yes & c.1049G >A & p.R350Q & $\begin{array}{c}\text { ZA } \\
\text { (VWD1) }\end{array}$ & Flat & Mild & 25.9 & vUS* & $\begin{array}{l}\text { PM1, PM2, } \\
\text { PP3 }\end{array}$ & 0.00000824 & - & This study \\
\hline 3 & HL0133 & F & 0 & 2 & No & No & c. $1049 \mathrm{G}>\mathrm{A}$ & p.R350Q & $\begin{array}{c}\text { ZA } \\
\text { (VWD1) }\end{array}$ & Flat & Moderate & 25.9 & VUS* & $\begin{array}{l}\text { PM1, PM2, } \\
\text { PP3, BP4 }\end{array}$ & 0.00000824 & - & This study \\
\hline 4 & HL0770 & $\mathrm{F}$ & 0 & 33 & No & Yes & c. $1424 \mathrm{C}>\mathrm{T}$ & p.P475L & $\begin{array}{c}\text { ZA } \\
\text { (VWD1) }\end{array}$ & Mid & Severe & 23.4 & VUS* & $\begin{array}{c}\text { PM1, PM2, } \\
\text { PP3, BS4, BP5 }\end{array}$ & 0.00000824 & - & This study \\
\hline 5 & HL2846 & M & 2 & 71 & No & No & c. $1685 \mathrm{C}>\mathrm{T}$ & p.T562M & $\mathrm{ZA}$ & Mid & Severe & 25.6 & VUS* & $\begin{array}{c}\text { PM1, PP3, } \\
\text { PP5 }\end{array}$ & 0.000099 & 0.0001 & Hildebrand, 2011 [22]. \\
\hline 6 & HL3080 & $\mathrm{F}$ & 60 & 73 & No & Yes & c.2093T $>C$ & p.V698A & $\mathrm{ZA}$ & Flat & Moderate & 14.1 & VUS & $\begin{array}{c}\text { PM1, PM2, } \\
\text { BP4 }\end{array}$ & - & 0.0001 & This study \\
\hline 7 & HL2684 & $\mathrm{F}$ & 6 & 10 & No & Yes & $c .2228 \mathrm{G}>\mathrm{T}$ & p.C743F & $\begin{array}{c}\text { ZA } \\
\text { (VWD2) }\end{array}$ & Mid & Mild & 24.4 & VUS* & $\begin{array}{c}\text { PM1, PM2, } \\
\text { PP3, BS2, BS4 }\end{array}$ & - & 0.0003 & This study \\
\hline 8 & HL1091 & $\mathrm{F}$ & 5 & 42 & No & Yes & c. $2228 \mathrm{G}>\mathrm{T}$ & p.C743F & $\begin{array}{c}\text { ZA } \\
\text { (VWD2) }\end{array}$ & High & Profound & 24.4 & vUS* & $\begin{array}{c}\text { PM1, PM2, } \\
\text { PP3, BS2, BS4 }\end{array}$ & - & 0.0003 & This study \\
\hline 9 & HL4176 & F & 0 & 45 & No & Yes & $c .2228 \mathrm{G}>\mathrm{T}$ & p.C743F & $\begin{array}{c}\text { ZA } \\
\text { (VWD2) }\end{array}$ & Flat & Profound & 24.4 & VUS* & $\begin{array}{l}\text { PM1, PM2, } \\
\text { PP3, BS2, BS4 }\end{array}$ & - & 0.0003 & This study \\
\hline 10 & HL1937 & $\mathrm{F}$ & 45 & 77 & No & Yes & c. $3556 \mathrm{C}>\mathrm{T}$ & p.R1186W & $\begin{array}{c}\text { ZA } \\
\text { (VWD3) }\end{array}$ & High & Severe & 33.0 & VUS* & $\begin{array}{l}\text { PM1, PP3, } \\
\text { BS1, BS4 }\end{array}$ & 0.0009 & 0.0001 & This study \\
\hline 11 & 2271 & M & Unknown & 6 & Unknown & Unknown & c. $4198 \mathrm{C}>\mathrm{T}$ & p.H1400Y & $\mathrm{ZA}$ (TIL3) & Mid & Moderate & 25.0 & VUS* & $\begin{array}{c}\text { PM1, PP3, } \\
\text { BS2 }\end{array}$ & 0.0002 & 0.0016 & Moteki,2012 [24]. \\
\hline 13 & HL0280 & M & 0 & 9 & No & No & c. $5908 \mathrm{G}>\mathrm{A}$ & p.A1970T & $\mathrm{ZP}$ & Flat & Mild & 13.5 & $\begin{array}{l}\text { Likely } \\
\text { Benign }\end{array}$ & BS2, BP4 & 0.0000906 & 0.001 & This study \\
\hline
\end{tabular}

Nucleotide and protein positions of TECTA variants are according to RefSeq: NM_005422. M: Male, F: Female, AA: Amino acid, NIDO: Nidogen-like domain, ZA: Zonahesin-like domain,

VWC: Von Willebrand factor C domain, TIL: Trypsin inhibitor-like domain, VWD: Von Willebrand factor D domain, ZP: Zona pellucida domain, MAF: Minor allele frequency. ${ }^{*}$ ACMG

category indicates the evidence for each variant classification. 




Figure 1. Cont. 




Figure 1. Cont. 




Figure 1. Cont. 


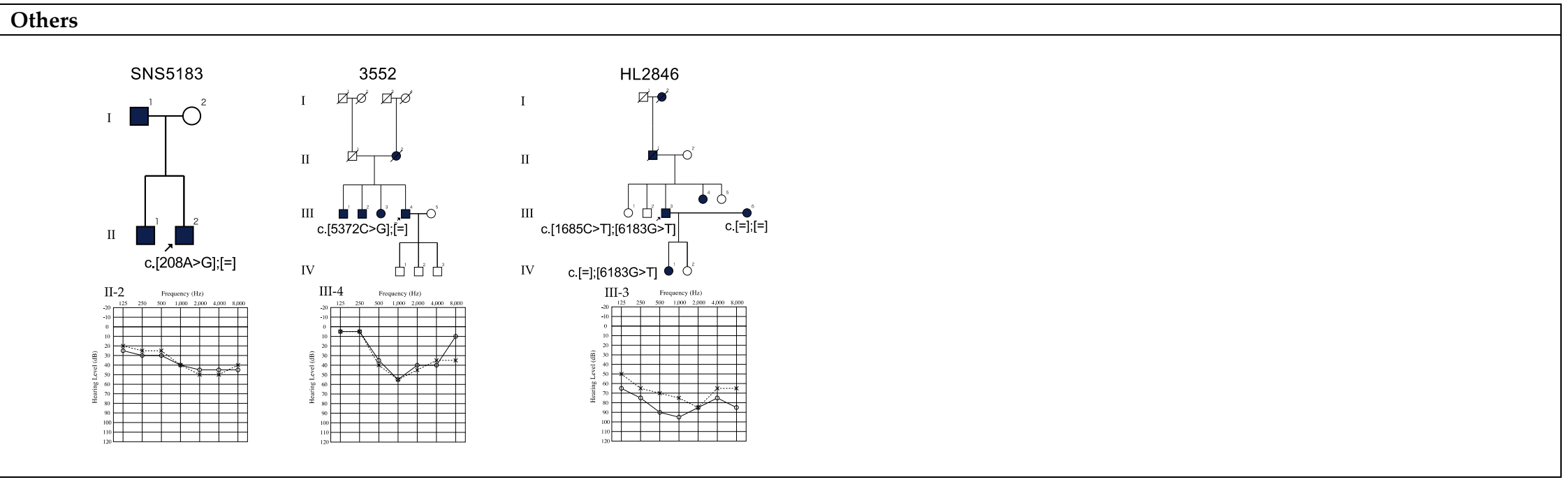

Figure 1. Pedigree and audiograms for each family with TECTA variants listed in Table 1. Arrows indicate the probands in each family. Genetic findings for each individual tested are noted in the pedigree. NIDO: nidogen-like domain, ZA: zonahesin-like domain, ZP: zona pellucida domain. Circle and solid line: right ear hearing threshold, X-mark and dotted line: left ear hearing threshold. 


\subsection{Genotype-Phenotype Correlation}

$\alpha$-tectorin has three major components: (1) a nidogen-like (NIDO) domain; (2) a large zonadhesin (ZA) domain containing three trypsin inhibitor-like (TIL) cysteine-rich domains, a von Willebrand factor type C (vWFC) and four von Willebrand factor type D (vWFD) domains; and (3) a zona pellucida (ZP) domain [29]. According to previous reports, mutations affecting the ZP domain are significantly associated with mid-frequency SNHL, whereas mutations in the ZA domain are associated with high-frequency SNHL $[5,7]$. Figure 2a shows a schema of the $\alpha$-tectorin domains, and overlapping pure-tone audiograms of individuals corresponding to the position of each domain.

a

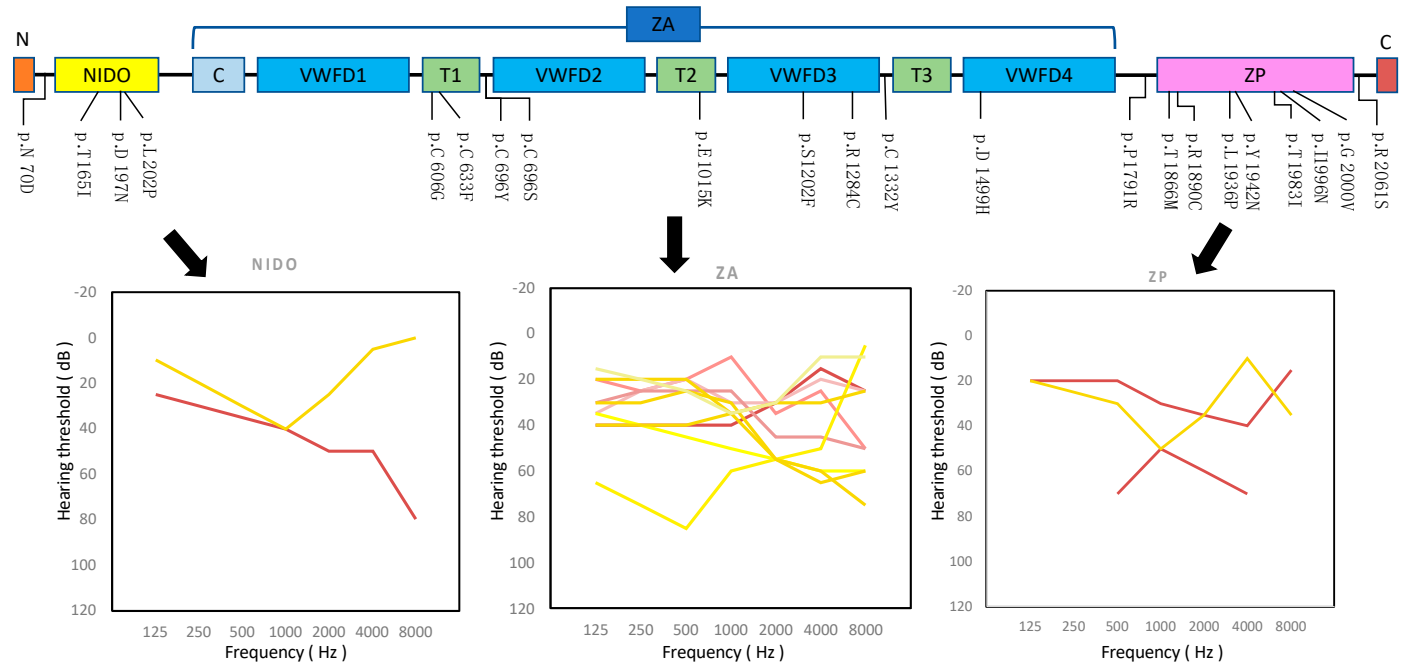

b
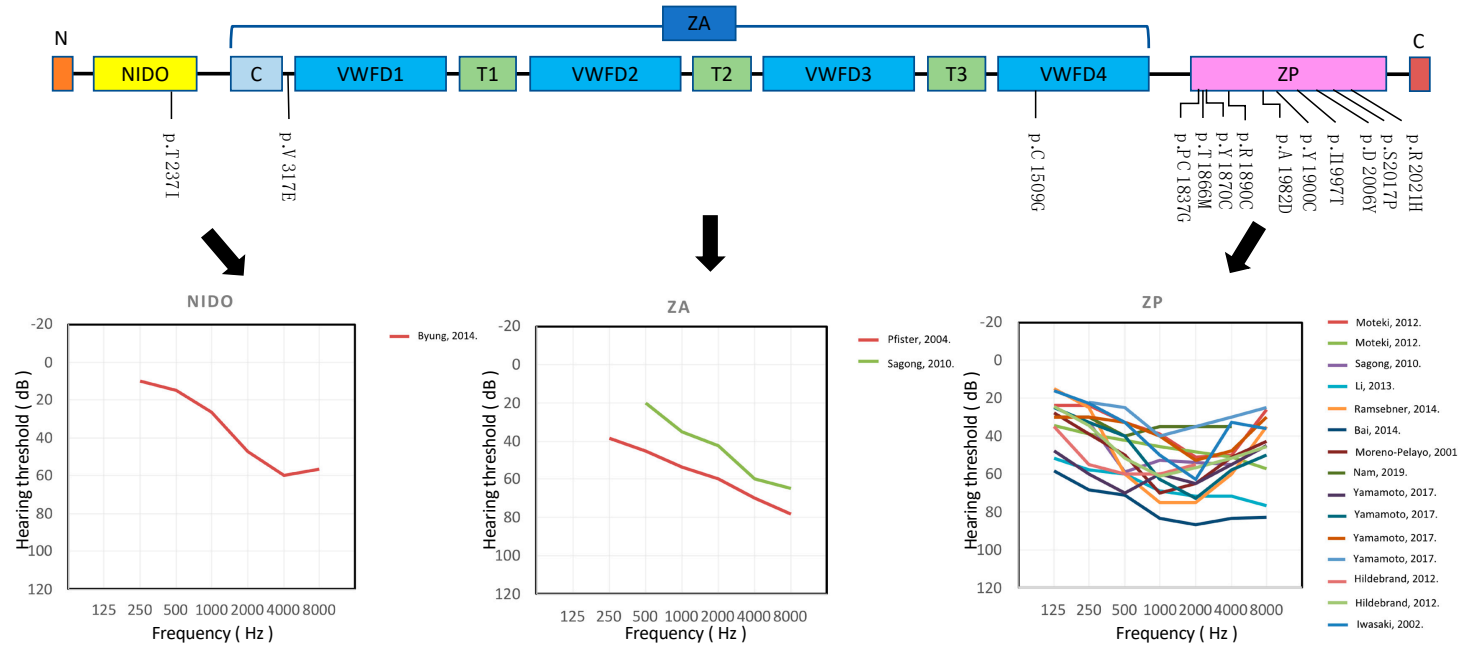

Figure 2. (a) Domain structure of $\alpha$-tectorin and the overlapping audiograms from the better hearing ear for the patients with each domain variant. Yellow lines indicate the candidate VUS variants, and red lines indicate the likely pathogenic variants. (b) The overlapping audiograms for the patients that were reported variants of TECTA in previously. NIDO: Nidogen-like domain, ZA: Zonahesin-like domain, C: Von Willebrand factor $\mathrm{C}$ domain, $\mathrm{T}(n)$ : Trypsin inhibitor-like domain (number), VWD: Von Willebrand factor D domain, ZP: Zona pellucida domain. 
In the NIDO domain, three variants, c.494C $>$ T, c.589G $>$ A and c.605T $>C$, were found in one family each. One variant, c.589G $>$ A, led to high-frequency SNHL. The other two variants, c.494C $>\mathrm{T}$ and c.605T $>$ C, led to mid-frequency SNHL.

In the ZA domain, the audiograms were varied, with mid-frequency HL observed in 6 subjects, flat-type HL in six subjects and high-frequency HL in eight subjects (Figure 2a).

In the ZP domain, seven of 12 variants were associated with mid-frequency $\mathrm{HL}$, and four variants were associated with flat HL. The severity of HL varied among the patients, however nine out of 11 cases had mild-moderate HL (Figure 2a).

\subsection{Relationship between Age and Hearing Levels}

To estimate the rate of hearing deterioration, we compared the age and hearing levels of 26 probands. As shown in Figure 3, each blue point indicated the average hearing level $(0.5-4 \mathrm{~K} \mathrm{~Hz})$ obtained at the time of blood sampling. In contrast, the red points are the average hearing level in the Japanese normal hearing population (age: 35-74 y). As a result, the rate of hearing deterioration in both groups was found to be the same and the regression lines for each group were parallel. Thus, hearing deterioration in TECTA patients was age-related and may not be accelerated by the gene mutation.



Figure 3. Detailed progression analysis of HL deterioration for patients with TECTA mutations. Lines indicate the linear regression for each group. Blue dots indicate the pure-tone average (PTA) of each patient with TECTA variants. Red dots indicating the PTA for Japanese normal hearing control population (Tsuiki et al. Audiology Japan 2003, 46, 235-240, in Japanese).

\subsection{The c.5597C>T (p.Thr1866Met) Variant in the ZP Domain}

One missense variant, c.5597C > T (p.Thr1866Met), in the ZP domain was identified in four unrelated families. The audiograms showed flat or mid-frequency HL, and were similar to the previously reported phenotype for variants located in the ZP domain [7].

We carried out haplotype analysis for these four families to determine whether this variant arose in a mutational hot spot or was a founder mutation. Table 3 shows the haplotype patterns within the $1-\mathrm{Mb}$ region surrounding this variant.

The different haplotypes observed suggest that this mutation occurred independently in each family. Thus, we suggest that c.5597C $>\mathrm{T}$ (p.Thr1866Met) arose from a mutational hot spot. 
Table 3. The haplotypes around the TECTA: c.5597C > T mutation identified from four families.

\begin{tabular}{|c|c|c|c|c|c|c|c|c|c|c|c|c|c|c|c|c|c|c|c|c|c|}
\hline \multirow{2}{*}{$\begin{array}{c}\text { Distance from the c.5597C }>\mathrm{T} \\
\text { Mutation } \\
(\mathrm{bp})\end{array}$} & \multirow{2}{*}{ Marker } & \multicolumn{2}{|c|}{$\begin{array}{c}\text { Fm1 } \\
\text { HL3681 }\end{array}$} & \multicolumn{2}{|c|}{$\begin{array}{c}\text { Fm2 } \\
\text { HL0605 }\end{array}$} & \multicolumn{8}{|c|}{ Fm3 } & \multicolumn{4}{|c|}{ Fm4 } & \multirow{2}{*}{\multicolumn{4}{|c|}{$\begin{array}{l}\text { Allele Frequency } \\
\text { (HapMap-JPT) }\end{array}$}} \\
\hline & & & & & & $\mathbf{A}$ & $\mathrm{U}$ & $\mathbf{A}$ & $\mathbf{U}$ & $\mathbf{A}$ & $\mathbf{U}$ & $\mathbf{A}$ & $\mathbf{U}$ & $\mathbf{A}$ & $\mathbf{U}$ & $\mathbf{A}$ & $\mathbf{U}$ & & & & \\
\hline 429809 & rs752979 & $\mathrm{T}$ & $\mathrm{C}$ & $T$ & $\mathrm{C}$ & $\mathrm{T}$ & $\mathrm{T}$ & 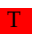 & $\mathrm{T}$ & $\mathrm{T}$ & $\mathrm{T}$ & C & $\mathrm{C}$ & $\mathrm{T}$ & $\mathrm{C}$ & $\mathrm{T}$ & $\mathrm{C}$ & $\mathrm{C}$ & 0.46 & $\mathrm{~T}$ & 0.54 \\
\hline 315340 & rs4430518 & $\mathrm{T}$ & G & $\mathrm{T}$ & $\mathrm{T}$ & G & G & $\mathrm{T}$ & $\mathrm{T}$ & $\mathrm{T}$ & $\mathrm{T}$ & $\mathrm{T}$ & G & $\mathrm{T}$ & $\mathrm{T}$ & $\mathrm{T}$ & $\mathrm{T}$ & G & 0.36 & $\mathrm{~T}$ & 0.64 \\
\hline 0 & c. $5597 \mathrm{C}>\mathrm{T}$ & 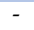 & & - & & - & & - & & - & & - & & - & & - & & & & & \\
\hline 589752 & rs7941422 & G & G & G & G & G & A & G & G & G & G & G & G & G & G & G & G & A & 0.17 & & 0.83 \\
\hline 649183 & rs621360 & $\mathrm{T}$ & C & $\mathrm{T}$ & $\mathrm{T}$ & $\mathrm{T}$ & C & $\mathrm{T}$ & $\mathrm{T}$ & $\mathrm{T}$ & $\mathrm{T}$ & $\mathrm{T}$ & $\mathrm{T}$ & C & $\mathrm{T}$ & C & C & C & 0.24 & & 0.76 \\
\hline 1089754 & rs489877 & A & A & A & $\mathrm{C}$ & A & A & A & A & A & A & A & A & A & C & A & A & A & 0.72 & C & 0.28 \\
\hline 1145749 & rs528219 & C & $\mathrm{C}$ & C & $\mathrm{T}$ & C & C & C & C & C & C & C & C & C & $\mathrm{T}$ & C & C & C & 0.69 & $\mathrm{~T}$ & 0.31 \\
\hline 1292838 & rs3906964 & A & A & G & G & G & A & G & A & G & A & G & G & G & G & G & A & A & 0.26 & G & 0.74 \\
\hline 1495732 & rs7117842 & $\mathrm{T}$ & $\mathrm{T}$ & $\mathrm{T}$ & $\mathrm{T}$ & $\mathrm{T}$ & C & $\mathrm{T}$ & C & $\mathrm{T}$ & C & $\mathrm{T}$ & C & $\mathrm{T}$ & C & $\mathrm{T}$ & C & C & 0.37 & $\mathrm{~T}$ & 0.63 \\
\hline
\end{tabular}

Red columns show the same region shared by unrelated families. Blue columns indicate different regions among families. Fm: Family, A: Putative affected allele, U: Putative unaffected allele. * (number): The family member with a TECTA mutation which is same variant to the each proband in Figure 1.

\section{Discussion}

In this study, we examined the prevalence of TECTA mutations in the Japanese ADSNHL population, and identified 26 TECTA-associated HL patients, which account for $3.2 \%$ of ADSNHL families. According to previous reports, TECTA mutations account for $2.9-5 \%$ of all ADSNHL patients $[24,30]$. Our result is consistent with those previous studies. The findings show that TECTA variants represent the second largest cause of ADSNHL in Japan, following KCNQ4 variants (6.6\%) [31].

In previous studies, only three mutations within the NIDO domain, c.632T >C (p.Phe211Ser), c.589G >A (p.Asp197Asn), and c.710C $>$ T (p.Thr237Ile), were reported in ADSNHL patients [32]. Furthermore, those cases showed mid- or high-frequency HL (Figure 2b) [32]. In our study, three variants, c.494C $>$ T, c.589G $>$ A, and c.605T $>C$, were identified in one family each, two of them novel. The patient with known c.589G $>$ A variant showed high-frequency HL, but the other patients (with the novel c.494C $>$ T and c.605T $>C$ variants) showed mid-frequency HL (Figure 2a). The c.589G $>$ A variant was reported previously, but that phenotype was mid-frequency SNHL [32]. Thus, the characteristic phenotype of HL was not observed in the NIDO domain variants. Previously, mutations in the vWFD2-D3 or vWFD4 repeat areas included in the ZA domain have been associated with high-frequency HL (Figure $2 b$ ). In this study, we identified 13 variants in the ZA domain region; however, various phenotypes were observed, so we couldn't identify any trend regarding HL in the ZA domain.

We also identified 11 variants in the ZP domain and most cases showed mid-frequency HL. This correlation between mutations in the ZP domain and mid-frequency HL has been reported previously (Figure 2b) $[9,17,19]$. This study is in agreement with the findings of those previous studies. In previous studies, some genes were reported as causative for mid-frequency HL, particularly, EYA4 (DFNA10), TECTA (DFNA8/12), COL11A2 (DFNA13), POU4F3 (DFNA15), and CCDC50 (DFNA44). Among those genes, mutations in TECTA are the most frequent cause of mid-frequency HL $[1,24,33,34]$. Yamamoto et al. reported that pathogenic and possibly pathogenic variants of TECTA were found in $6.0 \%$ of mid-frequency SNHL patients [35].

In this study, we focused on TECTA mutations identified in patients with ADSNHL as most of TECTA-associated HL is autosomal dominant and rarely autosomal recessive. The relationship between a domain structure and the inheritance mode of a TECTA mutation remains non conclusive. Regarding the type of mutation, missense mutations were predominantly observed in ADSNHL patients, while most loss of function mutations (nonsense, splicing, and frameshift mutations) in TECTA were identified in autosomal recessive cases. These results suggest that the mechanism of ADSNHL TECTA mutations is presumably dominant negative.

One of the clinical characteristics of the TECTA-associated HL was its non-progressive nature [18]. Byung et al. reported that the average HL deterioration rate didn't exceed $1 \mathrm{~dB} /$ year for the NIDO domain variant c.710C > T, p.Thr237Ile [32], and in another study, the p.Arg1890Cys mutation identified in a Dutch ADSNHL family, showed non-progressive, mid-frequency HL [9]. In this study, we 
analyzed the HL deterioration rate for TECTA-associated HL, and found it to be $0.35 \mathrm{~dB} /$ year. This HL deterioration rate is comparable to the hearing deterioration rate in a normal hearing control population. A previous report stated that patients with TECTA-associated mid-frequency HL might be prone to presbycusis as they are theoretically exposed to a lower level of sound energy as a result of cochlear amplification deficiency [36,37]. However, our results indicated that the hearing loss progression rate was the same as in the control group. Based on these results, we suggest that the mutations in the TECTA gene cause functional loss or malformation of the tectorial membrane and inhibit cochlear amplification. As a result of disturbed cochlear amplification, mild to moderate HL is observed. The HL progression ( $0.3 \mathrm{~dB}$ /year) observed in patients with TECTA mutations appears to reflect presbycusis, as the HL deterioration rate was comparable with that of normal hearing controls, suggesting that TECTA mutations do not accelerate HL deterioration.

In this study, one missense variant, c.5597C > T (p.Thr1866Met), in the ZP domain was detected in four unrelated families. This mutation was also reported in different populations including Americans, Spanish, and Koreans [22]. Hildebrand et al. performed haplotype analysis of the mutation found in the American and Spanish populations and found that they carried different haplotypes suggesting a unique founder effect could be identified in each population [22]. In this study, we also performed haplotype analysis for four families with this mutation and showed they carried different haplotypes even though all of them are Japanese. The results of this study and previous reports suggested that the c.5597C > T mutation arose in a mutational hot spot and occurs regardless of population.

\section{Conclusions}

The prevalence of TECTA mutations in Japanese ADSNHL families is estimated as 3.2\% (26/812 probands). Furthermore, when we limited our analysis to probands with 'Likely pathogenic' or 'Pathogenic' variants, it was found to be 1.8\% (15/812 probands). In the NIDO and ZA domain regions, various types of HL were observed, so we couldn't identify any trends. In the ZP domain, most cases showed mid-frequency HL. This result is in agreement with the findings of previous studies. The HL progression observed in the patients with TECTA mutations might reflect presbycusis, as the HL deterioration rate was comparable with that of normal hearing controls. A TECTA mutation itself is considered not to accelerate the HL deterioration. The recurrent c.5597C $>\mathrm{T}$ mutation might have arisen in a mutational hot spot and can be observed in many ethnic populations.

Supplementary Materials: The following are available online at http://www.mdpi.com/2073-4425/10/10/744/s1, Figure S1: Scatter plot of each identified variant's CADD score and higher MAF value in ToMMo 3.5kJPN or ExAC0.3., Table S1: The 68 target genes for hearing loss., Table S2: The in silico prediction scores for novel variants in this study.

Author Contributions: Conceptualization, H.M., S.-y.N. and S.-i.U.; methodology, H.M. and S.-y.N.; software, S.-y.N.; validation, R.Y.; formal analysis, R.Y. and S.-y.N.; investigation, S.-i.U.; resources, K.I., S.A., Y.H., M.H., R.M., T.I. (Tetsuo Ikezono), T.S., N.O., K.S., H.Y., T.I. (Takashi Ishino), K.O., T.I. (Tsukasa Ito), K.S.; data curation, R.Y., H.M. and S.-y.N.; writing—original draft preparation, R.Y.; writing-review and editing, R.Y., H.M. and S.-y.N.; visualization, R.Y.; supervision, S.-i.U.; project administration, S.-i.U.; funding acquisition, S.-i.U.

Funding: This research was funded by a Health and Labor Sciences Research Grant for Research on Rare and Intractable diseases and Comprehensive Research on Disability Health and Welfare from the Ministry of Health, Labor and Welfare of Japan (S.U. H29-Nanchitou(Nan)-Ippan-031), a Grant-in-Aid from Japan Agency for Medical Research and Development (AMED) (S.U. 16kk0205010h001, 18ek0109363h0001) and the Grant-in-Aid for Scientific Research (A) from the Ministry of Education, Culture, Sports, Science and Technology of Japan (S.U. 15H02565).

Acknowledgments: We thank the participants of the Deafness Gene Study Consortium for providing samples and clinical information. We also thank Sachiko Matsuda and Fumiko Tomioka for their technical assistance with this research.

Conflicts of Interest: The authors declare no conflicts of interest. The funders had no role in the design of the study; in the collection, analyses, or interpretation of data; in the writing of the manuscript, or in the decision to publish the results. 


\section{References}

1. Morton, C.C.; Nance, W.E. Newborn Hearing Screening_A Silent Revolution. N. Engl. J. Med. 2006, 354, $2151-2164$. [CrossRef] [PubMed]

2. Hereditary Hearing Loss Homepage. Available online: http://hereditaryhearingloss.org/ (accessed on 20 July 2019).

3. Schrijver, I. Hereditary non-syndromic sensorineural hearing loss: Transforming silence to sound. J. Mol. Diagn. 2004, 6, 275-284. [CrossRef]

4. Verhoeven, K.; Van Laer, L.; Kirschhofer, K.; Legan, P.K.; Hughes, D.C.; Schatteman, I.; Verstreken, M.; Van Hauwe, P.; Coucke, P.; Chen, A.; et al. Mutations in the human $\alpha$-tectorin gene cause autosomal dominant nonsyndromic hearing impairment. Nat. Genet. 1998, 19, 60-62. [CrossRef] [PubMed]

5. Plantinga, R.F.; De Brouwer, A.P.M.; Huygen, P.L.M.; Kunst, H.P.M.; Kremer, H.; Cremers, C.W.R.J. A novel TECTA mutation in a Dutch DFNA8/12 family confirms genotype-phenotype correlation. J. Assoc. Res. Otolaryngol. 2006, 7, 173-181. [CrossRef] [PubMed]

6. Legan, P.K.; Lukashkina, V.A.; Goodyear, R.J.; Kössl, M.; Russell, I.J.; Richardson, G.P. A targeted deletion in $\alpha$-tectorin reveals that the tectorial membrane is required for the gain and timing of cochlear feedback. Neuron 2000, 28, 273-285. [CrossRef]

7. Plantinga, R.F.; Cremers, C.W.R.J.; Huygen, P.L.M.; Kunst, H.P.M.; Bosman, A.J. Audiological evaluation of affected members from a Dutch DFNA8/12 (TECTA) family. J. Assoc. Res. Otolaryngol. 2007, 8, 1-7. [CrossRef] [PubMed]

8. Nishio, S.; Moteki, H.; Usami, S. Simple and efficient germline copy number variant visualization method for the Ion AmpliSeq ${ }^{\mathrm{TM}}$ custom panel. Mol. Genet. Genom. Med. 2018, 6, 678-686. [CrossRef] [PubMed]

9. Wang, K.; Li, M.; Hakonarson, H. ANNOVAR: Functional annotation of genetic variants from high-throughput sequencing data. Nucleic Acids Res. 2010, 38, e164. [CrossRef]

10. Abecasis, G.R.; Auton, A.; Brooks, L.D.; DePristo, M.A.; Durbin, R.M.; Handsaker, R.E.; Kang, H.M.; Marth, G.T.; McVean, G.A. An integrated map of genetic variation from 1,092 human genomes. Nature 2012, $491,56-65$.

11. NHLBI Exome Sequencing Project (ESP) Exome Variant Server. Available online: http://evs.gs.washington. edu/EVS/ (accessed on 15 May 2018).

12. Nakahara, M.; Higasa, K.; Nakamura, S.; Tabata, Y.; Kawaguchi, T.; Ishii, M.; Matsubara, K.; Matsuda, F.; Yamada, R. Large-scale East-Asian eQTL mapping reveals novel candidate genes for LD mapping and the genomic landscape of transcriptional effects of sequence variants. PLoS ONE 2014. [CrossRef]

13. Kumar, P.; Henikoff, S.; Ng, P.C. Predicting the effects of coding non-synonymous variants on protein function using the SIFT algorithm. Nat. Protoc. 2009, 4, 1073-1081. [CrossRef] [PubMed]

14. Adzhubei, I.A.; Schmidt, S.; Peshkin, L.; Ramensky, V.E.; Gerasimova, A.; Bork, P.; Kondrashov, A.S.; Sunyaev, S.R. A method and server for predicting damaging missense mutations. Nat. Methods 2010, 7, 248-249. [CrossRef] [PubMed]

15. Chun, S.; Fay, J.C. Identification of deleterious mutations within three human genomes. Genome Res. 2009, 19, 1553-1561. [CrossRef] [PubMed]

16. Schwarz, J.M.; Rödelsperger, C.; Schuelke, M.; Seelow, D. MutationTaster evaluates disease-causing potential of sequence alterations. Nat. Methods 2010, 7, 575-576. [CrossRef] [PubMed]

17. Reva, B.; Antipin, Y.; Sander, C. Predicting the functional impact of protein mutations: Application to cancer genomics. Nucleic Acids Res. 2011, 39, e118. [CrossRef] [PubMed]

18. Richards, S.; Aziz, N.; Bale, S.; Bick, D.; Das, S.; Gastier-Foster, J.; Grody, W.W.; Hegde, M.; Lyon, E.; Spector, E.; et al. Standards and guidelines for the interpretation of sequence variants: A joint consensus recommendation of the American College of Medical Genetics and Genomics and the Association for Molecular Pathology. Genet. Med. 2015, 17, 405-424. [CrossRef] [PubMed]

19. Li, Q.; Wang, K. InterVar: Clinical Interpretation of Genetic Variants by the 2015 ACMG-AMP Guidelines. Am. J. Hum. Genet. 2017, 100, 267-280. [CrossRef] [PubMed]

20. Kircher, M.; Witten, D.M.; Jain, P.; O'roak, B.J.; Cooper, G.M.; Shendure, J. A general framework for estimating the relative pathogenicity of human genetic variants. Nat. Genet. 2014, 46, 310-315. [CrossRef] [PubMed]

21. SNPinfo Web Server. Available online: https://snpinfo.niehs.nih.gov/ (accessed on 15 May 2018). 
22. Hildebrand, M.S.; Morín, M.; Meyer, N.C.; Mayo, F.; Modamio-Hoybjor, S.; Mencía, A.; Olavarrieta, L.; Morales-Angulo, C.; Nishimura, C.J.; Workman, H.; et al. DFNA8/12 caused by TECTA mutations is the most identified subtype of nonsyndromic autosomal dominant hearing loss. Hum. Mutat. 2011, 32, 825-834. [CrossRef] [PubMed]

23. Sagong, B.; Park, R.; Kim, Y.H.; Lee, K.Y.; Baek, J.I.; Cho, H.J.; Cho, I.J.; Kim, U.K.; Lee, S.H. Two novel missense mutations in the TECTA gene in Korean families with autosomal dominant nonsyndromic hearing loss. Ann. Clin. Lab. Sci. 2010, 40, 380-385.

24. Moteki, H.; Nishio, S.; Hashimoto, S.; Takumi, Y.; Iwasaki, S.; Takeichi, N.; Fukuda, S.; Usami, S. TECTA mutations in Japanese with mid-frequency hearing loss affected by zona pellucida domain protein secretion. J. Hum. Genet. 2012, 57, 587-592. [CrossRef] [PubMed]

25. ExAC Browser (Beta)-Exome Aggregation Consortium. Available online: http://exac.broadinstitute.org/ (accessed on 1 July 2019).

26. 5KJPN-Integrative Japanese Genome Variation Database. Available online: https://jmorp.megabank.tohoku. ac.jp/201905/ (accessed on 1 July 2019).

27. Mustapha, M. An alpha-tectorin gene defect causes a newly identified autosomal recessive form of sensorineural pre-lingual non-syndromic deafness, DFNB21. Hum. Mol. Genet. 1999, 8, 409-412. [CrossRef] [PubMed]

28. Kim, A.R.; Chang, M.Y.; Koo, J.W.; Oh, S.H.; Choi, B.Y. Novel TECTA mutations identified in stable sensorineural hearing loss and their clinical implications. Audiol. Neurotol. 2015, 20, 17-25. [CrossRef] [PubMed]

29. UniProt. Available online: https://www.uniprot.org/uniprot/O75443 (accessed on 25 July 2019).

30. Sloan-Heggen, C.M.; Bierer, A.O.; Shearer, A.E.; Kolbe, D.L.; Nishimura, C.J.; Frees, K.L.; Ephraim, S.S.; Shibata, S.B.; Booth, K.T.; Campbell, C.A.; et al. Comprehensive genetic testing in the clinical evaluation of 1119 patients with hearing loss. Hum. Genet. 2016, 135, 441-450. [CrossRef] [PubMed]

31. Naito, T.; Nishio, S.; Iwasa, Y.; Yano, T.; Kumakawa, K.; Abe, S.; Ishikawa, K.; Kojima, H.; Namba, A.; Oshikawa, C.; et al. Comprehensive Genetic Screening of KCNQ4 in a Large Autosomal Dominant Nonsyndromic Hearing Loss Cohort: Genotype-Phenotype Correlations and a Founder Mutation. PLoS ONE 2013, 8, e63231. [CrossRef] [PubMed]

32. Choi, B.Y.; Kim, J.; Chung, J.; Kim, A.R.; Mun, S.J.; Kang, S.I.L.; Lee, S.H.; Kim, N.; Oh, S.H. Whole-exome sequencing identifies a novel genotype-phenotype correlation in the entactin domain of the known deafness gene TECTA. PLoS ONE 2014, 9, e97040. [CrossRef] [PubMed]

33. Kitano, T.; Miyagawa, M.; Nishio, S.; Moteki,H.; Oda, K.; Ohyama, K.; Miyazaki,H.; Hidaka, H.; Nakamura, K.; Murata, T.; et al. POU4F3 mutation screening in Japanese hearing loss patients: Massively parallel DNA sequencing-34based analysis identified novel variants associated with autosomal dominant hearing loss. PLOS ONE 2017. [CrossRef] [PubMed]

34. Xia, W.; Liu, F.; Ma, D. Research progress in pathogenic genes of hereditary non-syndromic mid-frequency deafness. Front. Med. 2016, 10, 137-142. [CrossRef] [PubMed]

35. Yamamoto, N.; Mutai, H.; Namba, K.; Morita, N.; Masuda, S.; Nishi, Y.; Nakano, A.; Masuda, S.; Fujioka, M.; Kaga, K.; et al. Prevalence of TECTA mutation in patients with mid-frequency sensorineural hearing loss. Orphanet J. Rare Dis. 2017, 12, 157. [CrossRef] [PubMed]

36. Behlouli, A.; Bonnet, C.; Abdi, S.; Hasbellaoui, M.; Boudjenah, F.; Hardelin, J.P.; Louha, M.; Makrelouf, M.; Ammar-Khodja, F.; Zenati, A.; et al. A novel biallelic splice site mutation of TECTA causes moderate to severe hearing impairment in an Algerian family. Int. J. Pediatr. Otorhinolaryngol. 2016, 87, 28-33. [CrossRef]

37. Su, Y.; Tang, W.X.; Gao, X.; Yu, F.; Dai, Z.Y.; Zhao, J.D.; Lu, Y.; Ji, F.; Huang, S.S.; Yuan, Y.Y.; et al. A novel mutation in the TECTA gene in a Chinese family with autosomal dominant nonsyndromic hearing loss. PLOS ONE 2014. [CrossRef] [PubMed]

(C) 2019 by the authors. Licensee MDPI, Basel, Switzerland. This article is an open access article distributed under the terms and conditions of the Creative Commons Attribution (CC BY) license (http://creativecommons.org/licenses/by/4.0/). 